\title{
Efficient Parametric Identification Method for High Voltage Pulse Transformers
}

\author{
D. Aguglia \\ CERN, Geneva, Switzerland \\ S. Candolfi, P. Viarouge, J. Cros \\ LEEPCI Lab., Quebec, Canada
}

Keywords: High Voltage Pulse Transformers

\begin{abstract}
This paper presents a new identification method for a pulse transformer equivalent circuit. It is based on an analytical approximation of the frequency-domain impedance data derived from a no-load test with open-circuited secondary winding and only requires measurements of primary current and voltage without phase data. Compared with time consuming and complex methods based on off-line non-linear identification procedures, this simple method also gives an estimation of the error on the identified parameters. The method is validated on an existing pulse transformer.
\end{abstract}

Presented at: IEEE pulsed power and plasma science conference (PPPS),

San Francisco, USA, June 2013

Geneva, Switzerland

November, 2014 


\title{
Efficient Parametric Identification Method for High Voltage Pulse Transformers
}

\author{
Sylvain Candolfi ${ }^{1}$, Member, IEEE, Davide Aguglia ${ }^{2}$, Member, IEEE, \\ Philippe Viarouge ${ }^{1}$, Member, IEEE, Jérôme $\operatorname{Cros}^{1}$, Member, IEEE \\ ${ }^{1}$ LEEPCI Lab., Electrical and Computer Eng. Dept., Laval University, G1V 0A6 Quebec (QC), Canada \\ Emails: sylvain.candolfi.1@ulaval.ca, philippe.viarouge@gel.ulaval.ca, jerome.cros@gel.ulaval.ca \\ ${ }^{2}$ CERN - European Organization for Nuclear Research, Technology Dept., Electric Power Converter Group \\ CH-1211 Geneva 23, Switzerland Email: davide.aguglia@cern.ch
}

\begin{abstract}
This paper presents a new identification method for a pulse transformer equivalent circuit. It is based on a analytical approximation of the frequency-domain impedance data derived from a no-load test with open-circuited secondary winding and only requires measurements of primary current and voltage without phase data. Compared with time consuming and complex methods based on off-line non-linear identification procedures, this simple method also gives an estimation of the error on the identified parameters. The method is validated on an existing pulse transformer.
\end{abstract}

\section{INTRODUCTION}

Klystrons tubes are high-frequency and high-power amplifiers widely used in particle accelerators. In order to avoid the overheating of the tubes and waste of energy, klystrons are supplied with pulsed electrical power by modulators. Classical long pulse modulators are made of a capacitor bank, solid-state switches and a monolithic pulse transformer. The capacitor bank is constantly charged to smooth the power fluctuations on the electrical network side and quickly discharged through the pulse transformer. The pulse transformer adapts the voltage to the specification of the klystron and allows the use of low voltage solid-state switches on the primary side. The output pulse shape is very important for the performance of the klystron operation. The real pulse shape differs from an ideal one by its rising and falling times, overshoot and flattop stability. The electrical equivalent circuit parameters of the pulse transformer are very important for the modulator design and control process. The standard electrical equivalent circuit include the windings resistances, the magnetizing and leakage inductance and three lumped capacitances. The value of the elements of the equivalent circuit parameters are usually predicted during the design process with analytical relations or by using FEA techniques [2]. Non-linear identification techniques and specific tests in the frequency domain are also carried out to measure these parameters on pulse transformers [3], but these identification methods usually require several tests and complex identification procedures which deliver no specific estimation of the precision on the equivalent circuit elements.

The new identification method presented in this paper only requires a simple treatment of the frequency domain impedance measurements derived from a tranformer no-load test with open secondary winding. These data are analyzed to select four specific frequencies and their related impedance values. All transformer equivalent circuit parameters can be derived from analytic approximations of these characteristic impedance values except the primary winding capacitance. This capacitance cannot be determined because it is small compared to the other capacitances, but this parameter is not critical for the modulator design. The parameter identification errors are related to the instrumentation precision and to the approximation of the method itself. This error can be estimated and quantified for a range of typical transformers. The equivalent circuit is used during the design process and equivalent circuit referred to primary is used to demonstrate the identification method. It is demonstrated that those circuits are equivalent under some conditions between the parameters of both circuits. Some relations between the characteristic impedance and response to a pulse is established. Finally a case study is presented on an existing transformer. The equivalent model of the transformer is deduced from a frequency-domain identification. The time-response to a pulse of the transformer and of the equivalent model are compared.

\section{EQUIVALENT PULSE TRANSFORMER MODEL}

The standard equivalent circuit [4] of pulse transformers is made of one magnetizing inductance, two leakage inductances, the resistances of the primary and secondary windings and three lumped capacitances (Fig. 1). Primary and secondary windings are both connected to ground. The capacitances take into account the energy stored in the electrical field and they have an important influence on the dynamic performance of the transformer. The values of the capacitances referred to primary side are derived by equaling the electrostatic energy of the equivalent circuit and equivalent circuit referred to primary [3]. The values of the capacitances referred to primary side are derived by equaling the electrostatic energy of the equivalent circuit and equivalent circuit referred to primary [3].

$$
\begin{gathered}
C_{11} V_{1}^{2}+C_{12}\left(V_{2}-V_{1}\right)^{2}+C_{22} V_{2}^{2}= \\
C_{11}^{\prime} V_{1}^{2}+C_{12}^{\prime}\left(a V_{2}-V_{1}\right)^{2}+C_{22}^{\prime}\left(a V_{2}\right)^{2}
\end{gathered}
$$

Because this relation must be true for any voltage $V_{1}$ and $V_{2}$, one can determine the equivalent capacitances referred to the primary side. 


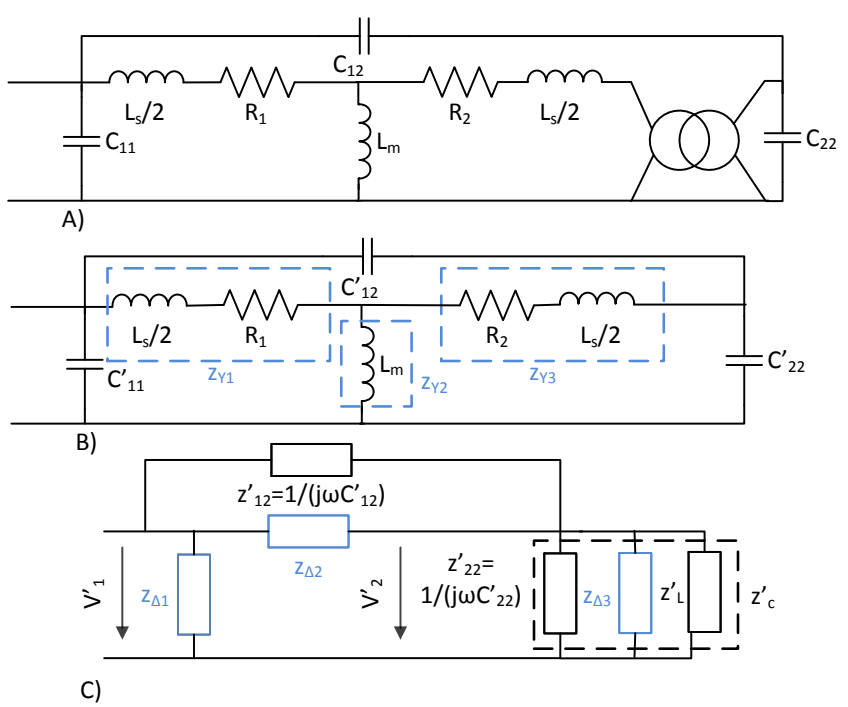

Fig. 1. A) Equivalent circuit B) Equivalent circuit referred to primary C) Circuit resulting of the Y- $\Delta$ transform

$$
\begin{aligned}
C_{11}^{\prime} & =C_{11}+C_{12} \frac{a-1}{a} \\
C_{22}^{\prime} & =\frac{C_{22}}{a^{2}}+C_{12} \frac{1-a}{a^{2}} \\
C_{12}^{\prime} & =\frac{C_{12}}{a}
\end{aligned}
$$

These equivalence lead to four observations:

- In the equivalent circuit, when both winding are supplied with their rated voltage, the voltage across $C_{12}$ terminals is much higher than the voltage across $C_{12}^{\prime}$ terminals for the same operation point. The energy stored in $C_{12}$ is distributed in the capacitances $C_{11}$, $C_{12}^{\prime}$ and $C_{22}$ of the equivalent circuit referred to the primary side.

- Naturally $C_{12}^{\prime}<C_{22}^{\prime}$ if $a<\frac{1}{2}$. This condition on the capacitance is mandatory for using the identification method.

- The secondary capacitance value referred to primary is amplified for a step-up transformer.

- the primary capacitance can be negative if the transformer ratio a is small. This characteristic illustrates the limit of the standard circuit modelling of the transformer but the equivalent circuit referred to primary must be considered as an equivalent mathematical representation of the transformer only.

By using these equivalent capacitances, one can obtain identical transfer functions $\frac{v_{2}}{v_{1}}$ and characteristic impedances $\frac{v_{1}}{i_{1}}$ for the equivalent circuit itself and its representation referred to the primary side. The demonstration can be greatly simplified by using a Y- $\Delta$ transformation on the equivalent circuit of the pulse transformer. The elements $z_{Y 1}, z_{Y 2}$ and $z_{Y 3}$ are transformed to $z_{\Delta 1}, z_{\Delta 2}$ and $z_{\Delta 3}$ ( Fig. 1). Because $z_{\Delta 1}$ is in parallel with $V_{1}$, it is not used for the evaluation of the transfer
TABLE I. EXPRESSION OF THE FREQUENCIES IN FUNCTION OF THE PARAMETERS

\begin{tabular}{|l|c|}
\hline Frequency & Expression \\
\hline$f_{0}$ & $\frac{R_{1}}{2 \pi L_{m}}$ \\
$f_{1}$ & $\frac{1}{2 \pi \sqrt{L_{m} C_{22}^{\prime}}}$ \\
$f_{2}$ & $\frac{1}{2 \pi \sqrt{L_{s} C_{22}^{\prime}}}$ \\
$f_{3}$ & $\frac{1}{2 \pi \sqrt{L_{s} C_{12}^{\prime}}}$ \\
\hline
\end{tabular}

function $\frac{V_{1}}{V_{2}^{\prime}} \cdot z_{\Delta 3}$ is placed in parallel with the impedance of the load $z_{L}^{\prime}$ to give the equivalent impedance $z_{c}^{\prime}$.

The transfer functions of the equivalent circuit and the equivalent circuit referred to the primary side can now be established:

$$
\begin{aligned}
& \frac{V_{1}}{V_{2}^{\prime}}=\frac{z_{12}^{\prime} z_{\Delta 2}+z_{22}^{\prime} z_{12}^{\prime}+z_{22}^{\prime} z_{\Delta 2}}{z_{22}^{\prime} z_{12}^{\prime}+z_{22}^{\prime} z_{\Delta 2}} \\
& \frac{V_{1}}{V_{2}}=\frac{z_{12} z_{\Delta 2}+z_{22} z_{\Delta 2}+a^{2} z_{12} z_{22}}{z_{22} z_{\Delta 2}+a z_{22} z_{12}}
\end{aligned}
$$

The impedances involving the capacitance of the transformer are replaced with their expression and the capacitances referred to primary are expressed from the capacitances of the equivalent circuit (eq. 2 to 4).

For both circuits the same transfer function is obtained.

$$
\frac{V_{1}}{V_{2}^{\prime}}=\frac{V_{1}}{a V_{2}}=\frac{j \omega z_{\Delta 2} z_{c}\left(C_{22}+C_{12}\right)+z_{\Delta 2}+a^{2} z_{c}}{z_{\Delta 2} z_{c} j \omega C_{12}+a z_{c}}
$$

For the transfer functions to be equal, the electrostatic energy between the equivalent circuit and equivalent circuit referred to primary must be equal (eq. 1).

The equivalence of the characteristic impedance between the circuits is demonstrated with the help of this last result. The energy in the circuit is the sum of the magnetic, electric and thermally dissipated energy. Secondary voltage being determined by the same transfer function for an identical primary voltage, the total energy is the same in both circuits (eq. 1). Consequently the consumed power are identical.

$$
P=\frac{d E}{d t}=V_{1} i_{1}=V_{1} i_{1}^{\prime}
$$

Primary voltages are the same for both circuits therefore currents $i_{1}$ and $i_{1}^{\prime}$ are identical (eq. 8). It follows that the impedances are equal.

\section{IMPEDANCE ANALYSIS OF THE EQUIVALENT MODEL}

The modulus of impedance $\mathrm{Z}$ is expressed in $d B_{\Omega}$, defined as $d B_{\Omega}=20 \log (|Z|)$. The impedance v.s. $\log (f)$ of individual resistance, inductance or capacitance in function of the frequency are represented by straight lines. The impedance of a typical pulse transformer (table II) is presented as well as the impedance of its elements referred to the primary side (Fig. 3). During a frequency sweep, each range of frequency sequentially approximates the impedance of one element. This method only requires measurement of the current and voltage modulus regardless to the phase. The requirements on instrumentation is less severe. When the difference of modulus between two impedances is large, the resulting impedance is 


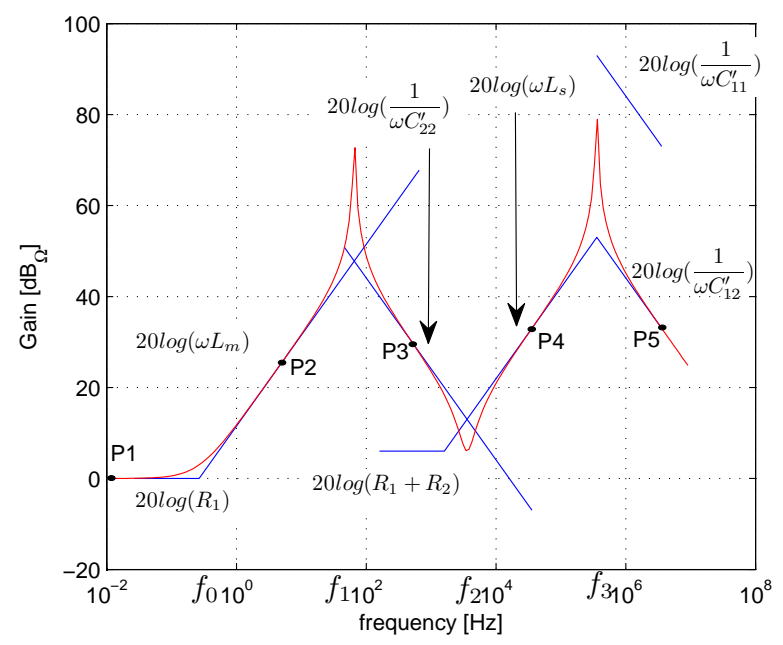

Fig. 2. Modulus of the characteristic impedance of the typical pulsed transformer referred to the primary (red) and impedance of the elements individually (blue)

around the bigger one if they are in series or the smallest one if they are in parallel. The resonances and anti-resonances occur when the modulus of impedance of two specific elements are equal. The expression of those frequencies is listed in Table I.

Below $f_{0}$ (Fig. 2) the capacitance can be considered as open circuits and inductances like short-circuits. The impedance is dominated by the primary winding resistance only. The primary winding resistance can be derived from a simple DC test. Above the frequency $f_{0}$, the magnetizing impedance modulus increases with frequency and becomes larger than the resistance of the primary winding which can be then neglected. The measured impedance is approximately the magnetizing impedance. At the frequency $f_{1}$ the magnetizing inductance and the secondary winding capacitance make a parallel resonance. Above this frequency the impedance modulus of the secondary winding capacitance becomes smaller than the impedance modulus of the magnetizing impedance. The capacitance is in parallel with the inductance therefore the inductance is neglected above $f_{1}$. At the frequency $f_{2}$ the leakage inductance and the secondary winding capacitance anti-resonate in series. The resulting impedance is the sum of the winding resistances because inductance and capacitance impedances are in phase-opposition. Above this frequency the impedance modulus of the leakage inductance becomes larger than the secondary winding impedance which can be considered as a short-circuit. Finally at the frequency $f_{3}$ the leakage inductance resonates with the inter-winding capacitance $C_{12}^{\prime}$. Above this frequency the impedance of the transformer is approximately equal to the impedance of the inter-windings capacitance if the primary capacitance is negligible. The order of the frequencies determines the shape of the impedance vs frequency characteristic. Some specific conditions on the transformer parameters are necessary to validate the method proposed in this paper.

- The magnetizing inductance must be greater than the leakage inductance: $L_{m}>L_{s}$

- The secondary winding capacitance must be bigger

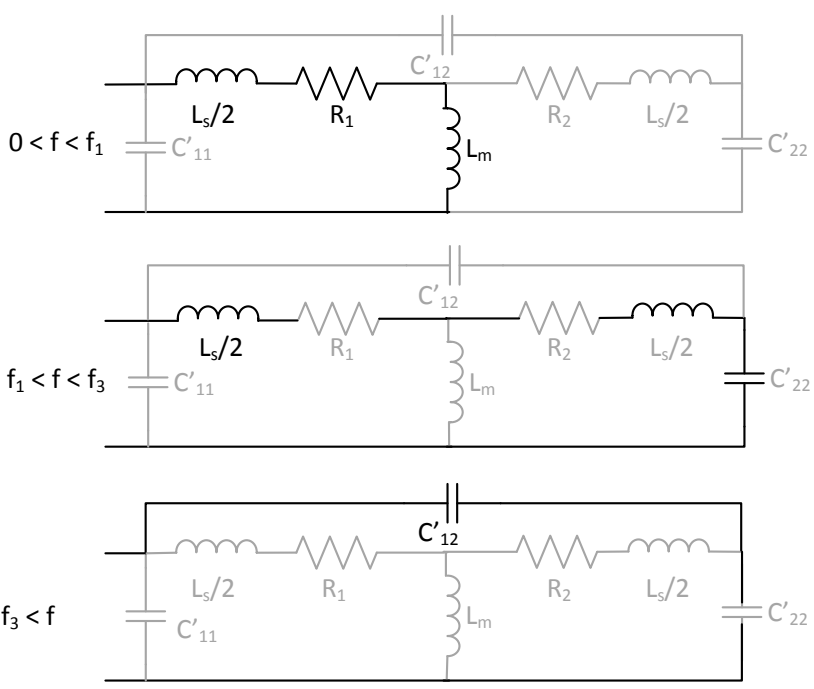

Fig. 3. Consecutive RL, RLC and C circuits in bolt approximating the impedance for each range of frequency.

TABLE II. TYPICAL VALUE OF THE PARAMETERS OF A $800 \mu$ S PULSE TRANSFORMER WITH A SECONDARY VOLTAGE OF $110 \mathrm{KV}$

\begin{tabular}{|l|c|c|}
\hline Parameter & Typical value & Unit \\
\hline$R_{1}$ & 1 & $\Omega$ \\
$R_{2}$ & 1 & $\Omega$ \\
$L_{s}$ & 200 & $\mu H$ \\
$L_{m}$ & 0.6 & $H$ \\
$C_{11}^{\prime}$ & 10 & $p F$ \\
$C_{22}^{\prime}$ & 10 & $\mu F$ \\
$C_{12}^{\prime}$ & 1 & $n F$ \\
\hline
\end{tabular}

than the inter-winding one referred to primary: $C_{22}^{\prime}>C_{12}^{\prime}$.

The first condition is verified as the permeability of magnetic core is very high compared to air. The second condition is true on transformers with a transforming ratio smaller than $\frac{1}{2}$ (eq. 3 and 4 ).

\section{PROPOSED IDENTIFICATION METHODOLOGY}

The identification method follows 7 steps:

1) Measurement of the characteristic impedance of the transformer with no-load from the primary.

2) Measurement of the resonant and cut-off frequencies.

3) Computation of the logarithmic average (mid. point) between two consecutive frequencies.

4) Measurement of the impedance at the computed frequencies, highest possible frequency and DC.

5) The impedances measured at these frequencies correspond to the impedances of the elements of the equivalent model (Table III).

6) The parameters referred to primary are retrieved from the impedances.

7) Estimation of the error from analysis of the plots (section VI). 
TABLE III. FREQUENCY AND CORRESPONDING MEASURED ELEMENT

\begin{tabular}{|c|c|c|}
\hline Point & Frequency $\mathrm{f}$ & Impedance measured \\
\hline$\overline{\mathrm{P} 1}$ & Continuous & $R_{1}$ \\
\hline P2 & $10 \frac{\log f_{0}+\log f_{1}}{2}$ & $2 \pi f\left(L_{m}+\frac{L_{s}}{2}\right)$ \\
\hline P3 & $10^{\frac{\log f_{1}+\log f_{2}}{2}}$ & $\frac{C_{22}^{\prime}}{2 \pi f}$ \\
\hline P4 & $f_{2}$ & $R_{1}+R_{2}$ \\
\hline P5 & $10^{\frac{\log _{J_{2}}+\log _{J_{3}}}{2}}$ & $2 \pi f L_{s}$ \\
\hline P6 & $10 f_{3}$ & $\frac{C_{12}}{2 \pi f}$ \\
\hline
\end{tabular}

\section{TIME-DOMAIN ILLUSTRATION OF THE PROPOSED METHOD VALIDITY RANGE}

Three poles and one zero have been identified in the characteristic impedance of a pulse transformer. These poles and zero can be related to the step-response of the transformer represented by its equivalent circuit. In the same way of the analytic approximation of the impedance, the impulse response without load is approximated by considering two circuits approximating the real one in order to handle only circuits of the $2^{\text {nd }}$ order or less. A resistive load would mainly damp the response of the transformer. The first circuit (Fig. $4 \mathrm{~A}$ ) is made of the capacitance, leakage inductance and winding resistances. Only the magnetizing inductance is neglected which only has influence at low frequency like the characteristic impedance demonstrates. The magnetizing inductance can be neglected at the beginning of the pulse. Its transient response to an impulse is the response of a serie $\left(R_{1}+R_{2}\right) L_{s}\left(C_{22}^{\prime}+C_{12}^{\prime}\right)$ circuit. In the under damped case the frequency of oscillation $f_{d}$ is:

$$
2 \pi f_{d}=\sqrt{\omega_{0}^{2}-\alpha^{2}}
$$

with

$$
\begin{aligned}
\omega_{0} & =\frac{1}{\sqrt{L_{s}\left(C_{22}^{\prime}+C_{12}^{\prime}\right)}} \\
\alpha & =\frac{\left(R_{1}+R_{2}\right)}{2 L_{s}}
\end{aligned}
$$

Neglecting $\alpha$ in front of $\omega_{0}$ we have a relation between the frequencies $f_{2}$ and $f_{3}$ of the impedance and the frequency of the oscillation.

$$
f_{d}^{2}=\frac{1}{\frac{1}{f_{2}^{2}}+\frac{1}{f_{3}^{2}}}
$$

The amplitude of the oscillation is given by the initial condition. At $\mathrm{t}=0$ the output voltage is given by the tension of the capacitive voltage divider formed of the capacitance $C_{12}^{\prime}$ and $C_{22}^{\prime}$. The ratio $\frac{C_{22}^{\prime}}{C_{12}^{\prime}}={\frac{f_{3}}{f_{2}}}^{2}$ determine the amplitude of the oscillation. If it is bigger than 1 then $f_{3}>>f_{2}$ and the amplitude is small. The condition on the capacitance for the application of the identification method is fulfill the amplitude of oscillation in the step-response is low.

The second circuit (Fig. 4 B) constituted of the inductances and resistances approximates the response of the transformer after a time $t>\alpha^{-1}$ when the ripple is damped. The capacitance are neglected as they have no influence at low frequency. The step-response is an exponential decay with a time constant $\tau$ related to the frequency $f_{0}$.

$$
\tau=\frac{1}{f_{0}}
$$
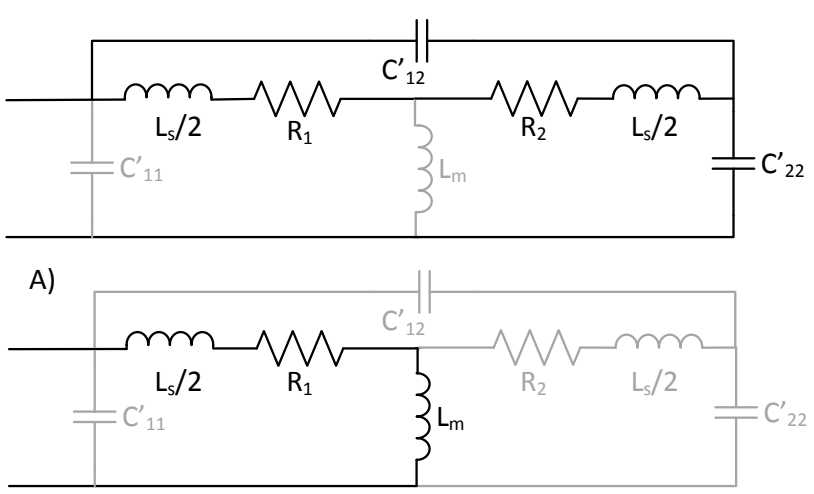

B)

Fig. 4. Circuits used for the approximation of the time-response of the transformer in the beginning of the pulse A) and at the end of the pulse B)

\section{A PRIORY ERROR ESTIMATION ON IDENTIFIED PARAMETERS}

The error between the measurement and the real equivalent parameter assuming the instruments have no uncertainty is evaluated with simulations. The error is presented in function of the frequencies in order to provide a direct link with the measured characteristic impedance. The error is mostly dependent on the two frequencies adjacent to the measurement point which include three parameters. The error in function of two variable parameters is computed, the third parameters is fixed and the others take the value of a typical pulse transformer (Table II). The values of the parameters are transformed into the frequencies (Table I). The third parameter is set to a new value and the error in function of the two other parameters is computed again. For each set of frequencies two errors are computed and the largest is retained to plot the graph and the bounds of the third parameter are always specified.

\section{A. Magnetizing inductance}

The magnetizing impedance is measured between the frequencies $f_{0}$ and $f_{1}$. The closer those two frequencies are, the bigger the error will be (Fig. 5 b)). The case where $f_{0}=f_{1}$ is the lower right limit of the plot. If there is more than two decades between the frequencies $f_{0}$ and $f_{1}$ the error on the inductance is less than $10 \%$. In all the cases presented the inductance is overestimated due to the proximity of the resonance with the secondary capacitance and to the primary winding resistance which increase the impedance at the measurement point. The upper left bound on the chart is due to the frequency $f_{2}$ becoming higher than $f_{3}$. The frequencies $f_{2}$ and $f_{3}$ have little influence on the error on $L_{m}$ as they do not influence the value of the characteristic impedance between $f_{0}$ and $f_{1}$ (Fig. 5 a)).

\section{B. Capacitance $C_{22}^{\prime}$}

The error on the secondary winding capacitance is near $0 \%$ for a large range of frequencies $f_{1}$ and $f_{2}$ (Fig. ??) thanks to the position of the measurement point between a resonance and an anti-resonance frequency. The error increase above $10 \%$ if the frequencies $f_{1}$ and $f_{2}$ are closer than 0.2 decade because of the proximity of the measurement point with the resonance 

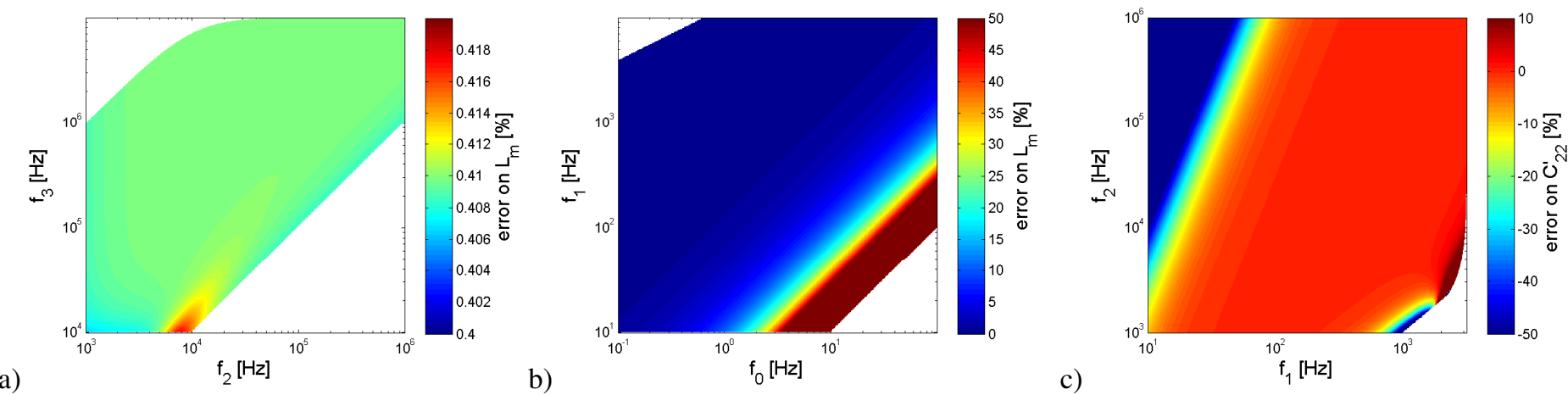

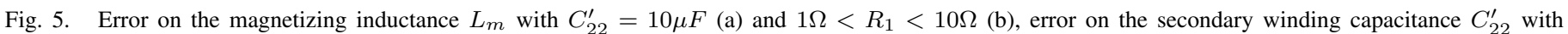
$10 \mu H<L_{m}<10 H$ (c) versus the resonant, anti-resonant and cut-off frequencies.

and anti-resonance. The error increase above $10 \%$ as well if the frequencies $f_{1}$ and $f_{2}$ are spaced by more than 2.8 decades. In this case the anti-resonance has a low quality factor and the measurement point is found on the anti-resonance (Fig. $6 \mathrm{c}$ )). This situation can be visually identified and solved by taking a measurement point where the impedance has a slope of 20 $\mathrm{dB} /$ decade.

\section{Leakage inductance}

There are several similarities between the measurement of the leakage inductance and of the secondary winding capacitance as both are measured between a resonance and an antiresonance frequency. If the frequencies $f_{2}$ and $f_{3}$ are close, the resonance lower the impedance, the leakage inductance is overestimated (Fig. 6 a)). The error is under $15 \%$ if $f_{2}$ and $f_{3}$ are separated by more than half-a-decade.

\section{Capacitance $C_{12}$}

The error on the inter-winding capacitance is under $5 \%$ for a large range of frequencies. If $f_{3}$ is high, the inter-wining capacitance is small and the primary capacitance cannot be neglected as it can be seen in the top right corner (Fig. 6 b)). If $f_{2}$ is close to $f_{3}$ then $C_{22}^{\prime} \approx C_{12}^{\prime}$ and $C_{22}^{\prime}$ cannot be neglected above $f_{3}$ but is in serie with $C^{\prime} 12$

\section{EXPERIMENTAL VALIDATION}

The method is tested on a prototype with primary and secondary windings on one leg (Fig. 7). The impedance is measured (Fig. 8). The shape of the impedance indicates that the criteria are fulfilled to use the method. The value of the impedance between the resonance frequencies are measured and the corresponding elements deduced (table III). The interwinging capacitance must be carefully measured. It must be done at the highest possible frequency allowed by the instruments to keep away the resonance but experimentally other high-frequency resonances which are not modeled by the lumped parameter model appear at very high frequency. A trade off between those resonances must be found.

The frequencies are evaluated, the measurement points found and the parameters computed (table IV). The characteristic impedance is simulated with these parameters and compared with the experimental one. Both are in concordance

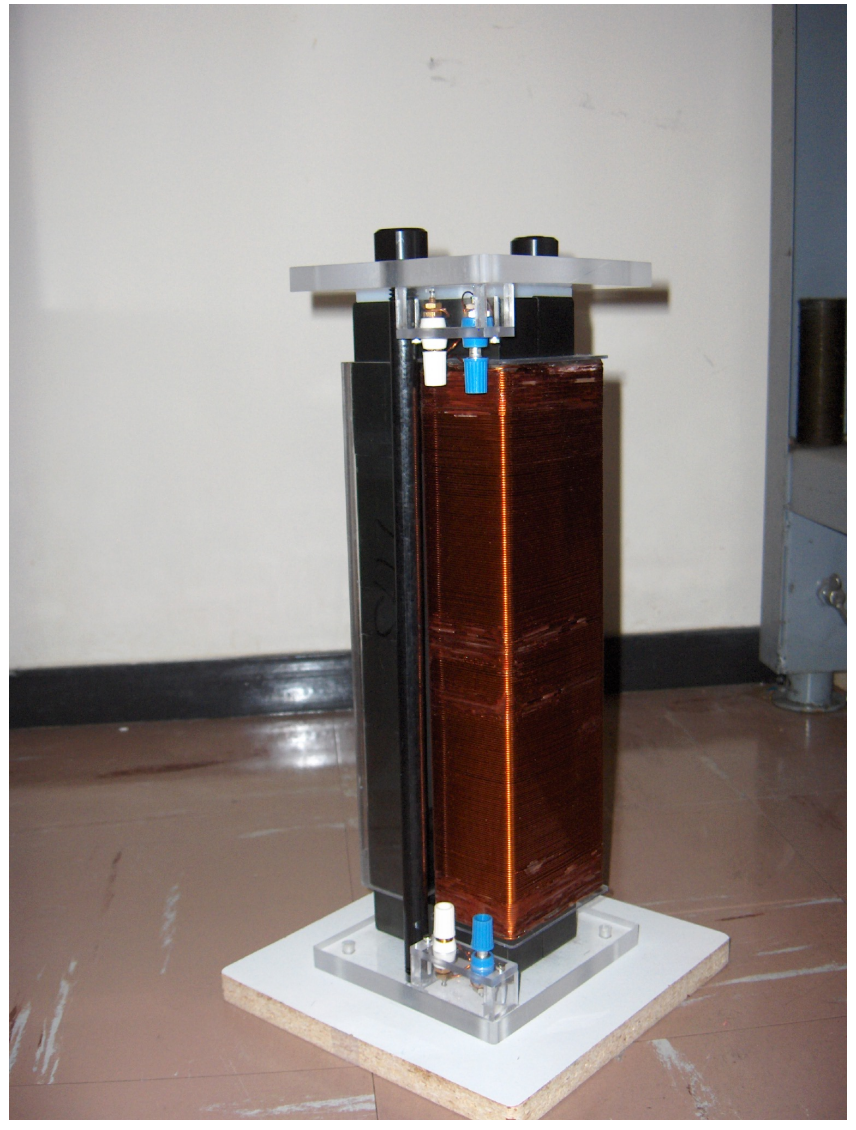

Fig. 7. Transformer prototype experimentally identified

at the measurement points and the error is small elsewhere. Neglecting the instrumentation incertitude, the error on the parameters can be evaluated on the plots ( Fig. 5 and 6) as all the measured parameters are within the limits adopted to plot the errors. The error on the magnetizing inductance and secondary winding capacitance are less than 5\% and less than $10 \%$ for the leakage inductance and inter-windings capacitance. An independent test is performed on the transformer. The response of the transformer to a pulse is recorded and simulated with the parameters of the equivalent model previously determined. Simulation and measurement are compared. 

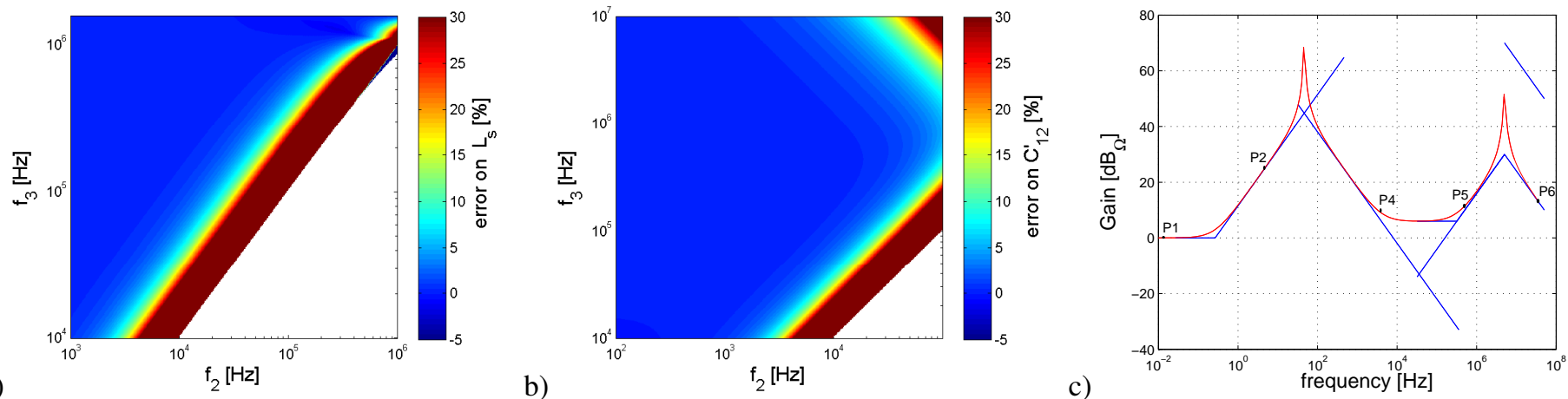

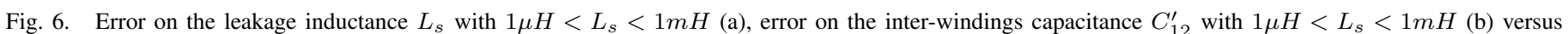
the resonant and anti-resonant frequencies and plot of the characteristic impedance in the unfavourable case where $f_{1}=45 \mathrm{~Hz}$ and $f_{2}=3.610^{4} \mathrm{~Hz}(\mathrm{c})$.
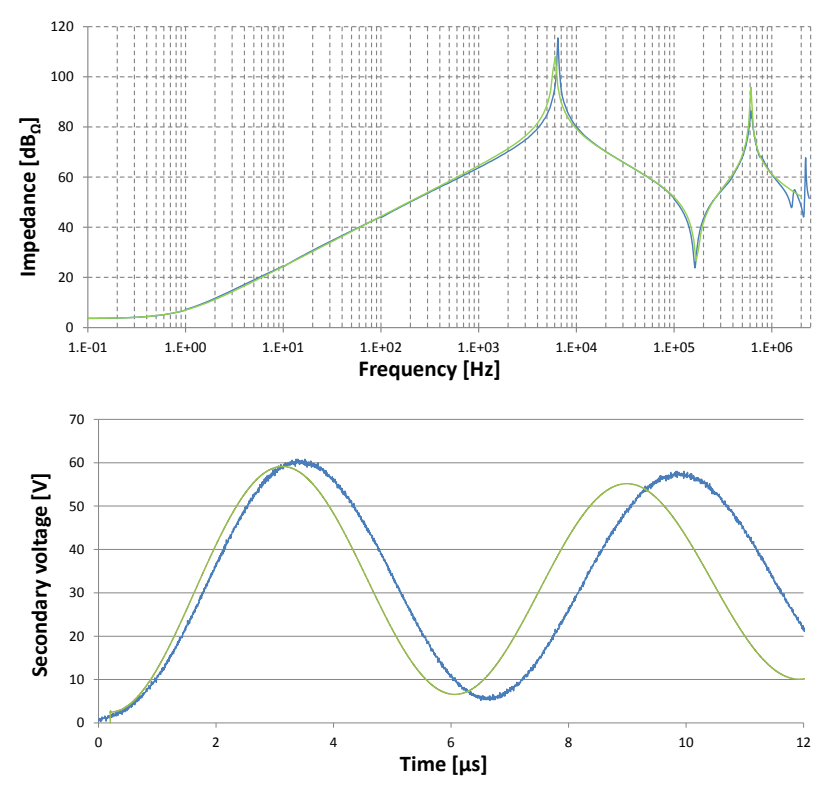

Fig. 8. Measure (blue) and simulation with the measured equivalent elements(green) of the characteristic impedance and time response to a step at the primary

TABLE IV. EXPERIMENTAL FREQUENCIES AND MEASURED ELEMENT

\begin{tabular}{|l|c|}
\hline Frequency & Value $[\mathrm{Hz}]$ \\
\hline$f_{0}$ & $9.610^{-1}$ \\
$f_{1}$ & $6.510^{3}$ \\
$f_{2}$ & $1.6410^{5}$ \\
$f_{3}$ & $6.210^{5}$ \\
\hline
\end{tabular}

\begin{tabular}{|l|c|c|}
\hline Element & value & Unit \\
\hline$R_{1}$ & 1.52 & $\Omega$ \\
$L_{m}$ & 262 & $m H$ \\
$C_{22}^{\prime}$ & 2.7 & $\mathrm{n} \mathrm{F}$ \\
$L_{s}$ & 297 & $\mu H$ \\
$C_{12}^{\prime}$ & 230 & $\mathrm{p} \mathrm{F}$ \\
\hline
\end{tabular}

In the simulated step-response the capacitance are instantly charged leading to a discontinuity. In the measured response this discontinuity disappears. The capacitances are charged through the resistance and inductance of the connecting wires, adding a charging time. The frequency of the simulated response is underestimated of about $10 \%$. This is inside the incertitude on the parameters. The amplitude of the ripple is well predicted.

\section{CONCLUSION}

It has been demonstrated that the frequency-domain impedance measurement derived from a no-load test with open-circuited secondary winding is sufficient to identify the equivalent circuit of a pulse transformer. In such a test, phase measurements are not necessary. For a large range of pulse transformers, the parameters are predicted with sufficient accuracy to simulate the time response of the transformer and the method has been validated on a prototype. The proposed method combines analytic approximations and numerical simulations to confirm the validity of the hypothesis. Further refinements can be achieved by taking into account the resonance frequencies or by taking the parameters derived with the proposed method as initial guess of a non-linear optimization identification procedure.

\section{ACKNOWLEDGMENT}

This work is financially and materially supported by CERN, Switzerland.

\section{REFERENCES}

[1] D. Aguglia, C. A. Martins, M. Cerqueira Bastos, D. Nisbet, D.Siemaszko, E. Sklavounou, P. Viarouge, Klystron Modulator Technology Challenges for the Compact Linear Collider (CLIC), IEEE Conference on Pulsed Power, Washington DC 2011, pp. 1-10

[2] P. Viarouge, D. Aguglia, C.A. Martins, J. Cros, Modelling and Dimensioning of High Voltage pulse Transformers for Klystron Modulators, $20^{\text {th }}$ International Conference on electrical machines (ICEM), 2-5 Sept. 2012, pp.2332-2338.

[3] D. Aguglia, P. Viarouge and A. Martins C., Frequency domain nonlinear identification method for high voltage pulse transformer, $20^{\text {th }}$ International Conference on electrical machines (ICEM), 2-5 Sept. 2012, pp.1977 - 1983.

[4] IEEE Standard for Pulse Transformers, ANSI/IEEE Std 390, 1987. 